\title{
Geometrical Data Validation in 3D Digital Cadastre - A Case Study for Victoria, Australia
}

\author{
Shojaei, D., Olfat, H., Faundez, S. I. Q., Kalantari, M., Rajabifard, A. \& Briffa, M.
}

\begin{abstract}
Data ambiguity and invalidity can cause significant expensive issues in the cadastral domain (e.g. legal disputes). An automated data validation can significantly help to reduce the potential issues. Quality assurance has been comprehensively investigated in various domains, however, the validation of 3D cadastral data is still in its early development. The availability of various regular and irregular shapes for 3D cadastral objects and modern building designs has resulted in a critical need for developing validation rules to ensure data validity and quality.

The land registry in Victoria, Australia, is investigating the technical requirements for implementing a 3D digital cadastre. The study of 3D cadastral data validation requirements has been part of this ongoing investigation. This study is being undertaken in three main phases including 1) developing geometrical validation rules, 2) developing non-geometrical validation rules, 3) implementing an online service to validate $3 \mathrm{D}$ cadastral data.

This paper aims to discuss the initial outcomes of the first phase of the aforementioned study which has focused on developing geometrical validation rules for 3D cadastral objects. The paper reviews the development of four geometrical validation rules which have been formalised using mathematical expressions to check the individual 3D parcels and their relationships with adjoining or neighbouring parcels. The first validation rule checks the compatibility of the cancelled parcel against the created parcels. The second rule deals with parcel collision detection which is required for flagging unacceptable intersection of 3D objects. The third rule ensures the faces forming a 3D parcel are flat. The fourth validation rule assures 3D objects are watertight. The paper concludes with a discussion around the impacts of the proposed validation checks on the subdivision process and future research for the Victorian 3D digital cadastre.
\end{abstract}

Keywords: cadastre, validation, geometry, 3D, ePlan, LandXML, Victoria.

\section{Introduction}

The density of our urban environments is increasing and the lack of available land has led decision makers to think about the proper use of spaces above and below the ground. Cadastral systems deal with these spaces and associated rights, restrictions and responsibilities (RRRs). In recent years, there has been a growing trend towards more efficiently using spaces. However, the current cadastres do not efficiently register and present complex ownership rights in mixed-use and high rise developments (Shojaei et al., 2013, Aien et al., 2015, Ho, et al., 2015). Shojaei (2014) has reported some of the main shortcomings of current cadastral systems.

A 3D digital cadastre is expected to facilitate the registration process (Aien et al., 2012), save time and cost, increase transparency in land and property transactions, and improve land use and management (Shojaei, 2014). For example, in a 3D digital cadastre, overlapped spatial units can be validated and geometries can be checked to ensure rights are protected and disputes are minimised. 
The State of Victoria has been moving towards a digital cadastre since the introduction of ePlan in this jurisdiction. ePlan is a collaborative program between the Australian land authorities and the surveying industry, in conjunction with ICSM ${ }^{1}$ which aims to replace paper and PDF plans with digital files (Olfat et al., 2016). In 2009, the ICSM endorsed the national ePlan as an agreed conceptual data model of a cadastral survey that meets the needs of the jurisdictions in Australia. In 2011, an ePlan Protocol was developed to map the components of the ePlan data model to LandXML, a specialised XML data file format containing civil engineering and survey measurement data commonly used in the land development and transportation industries ${ }^{2}$.

Following the release of the ICSM's strategy on Cadastre 2034, the ePlan Working Group has started to investigate the requirements for supporting 3D building subdivisions in ePlan. Cadastre 2034 Strategy has a vision to enable people to understand their RRRs related to land and property in a spatially accurate and 3D environment. This vision leads to changes in current subdivisional processes. From the process point of view, 3D data must be available to provide accurate information of the land and property. This often leads to new methods of data collection and sourcing. Having the required 3D data, the analysis and registration will give a better picture of RRRs (ICSM, 2015).

The emphasis is towards achieving a 3D digital cadastral system that enables the community and stakeholders such as councils, referral authorities, real estate agencies, insurance companies, developers, and architects to readily and confidently identify the location and related interests to land and property. The key element of this change is to ensure the efficiency of the cadastral system in Australian jurisdictions. This requires a strong commitment of stakeholders to improve the management and sharing of cadastral information and enhance their systems and infrastructures to enable this change (ICSM, 2015).

To facilitate this commitment in Victoria, Land Use Victoria (LUV) commenced investigating the technical aspect of a 3D digital cadastre in 2014 by looking at various topics including 3D data modelling, storage, validation, and visualisation. In the data modelling study, the LandXML data model was investigated in terms of supporting 3D cadastral objects and the most appropriate modelling approach was identified (Shojaei, et al., 2016). In the data storage study, various approaches for storing LandXML files are currently under investigation. As part of the data visualisation study, a 3D visualisation prototype ${ }^{3}$ was developed to showcase the concept of $3 \mathrm{D}$ cadastre and its benefits (Olfat et al., 2017). The initial outcomes of the 3D data validation study are also discussed in this paper.

Data validation controls the data quality and helps avoiding data issues in the future. It is to ensure the consistency, integrity, correctness and completeness of data (Wagner et al., 2013). Data validation is not a new topic and it has been investigated in different disciplines such as 3D City Models (Kazar et al., 2008, and Wagner et al., 2013), medical image processing (Gerig et al., 2001), and DBMS ${ }^{4}$ (Arens et al., 2005). In 3D cadastre, low data quality may cause confusion in interpreting the ownership boundaries which may cause problems during or after property registration (Aien et al., 2014). Therefore, 3D ownership rights must be correct and unambiguous, as an error or ambiguity in data can cause expensive legal disputes (Thompson and Van Oosterom, 2014).

In Victoria, there is a 2D ePlan validation service in place which identifies some of the errors and potential problems in plans at an early stage and allows the surveyor to correct or justify them prior to

\footnotetext{
${ }^{1}$ The Intergovernmental Committee on Surveying and Mapping

${ }^{2}$ www.landxml.org

${ }^{3}$ www.spear.land.vic.gov.au/spear/pages/eplan/3d-digital-cadastre/land-victoria-3d-eplan-prototype.shtml

${ }^{4}$ Data Base Management System
} 
the examination process. This will result in a reduction in the number of refusals and requisitions in the registration process. There are 128 ePlan validation rules supported by this service which cover three main areas of 'survey accuracy (e.g. parcel area, parcel observations closure)', 'survey examination rules (e.g. appropriate title connections)' and 'metadata completeness (e.g. easement purpose)'.

Validation rules for 3D cadastre can be classified into two main categories including non-geometrical and geometrical rules. Non-geometrical rules look at the semantics of 3D objects to support the business of cadastre. For example, the address of a parcel must be correctly captured. Semantic checks are easier to implement compared with geometrical ones. In contrast, geometrical rules look at the geometry of objects. Geometrical validation is more complicated in a 3D cadastral context compared to 2D cadastre due to the variety of possible shapes and geometries in space (Karki et al., 2010). These rules could be classified as internal and external rules. Internal rules assure objects are correctly defined. For example, is the boundary of an object complete and there is no gap? However, external rules assure objects have correct relationships to other neighbouring objects. For example, two 3D objects must not clash.

As part of the 3D cadastral data validation, a study with three phases has been designed and shown in Figure 1.

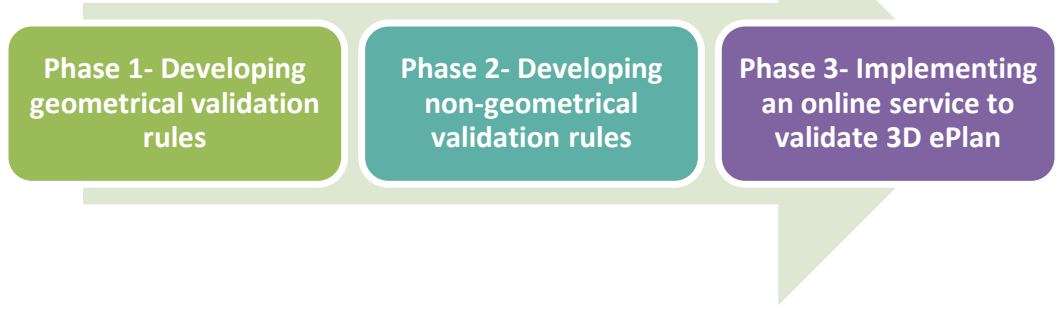

Figure 1. Phases of 3D cadastral data validation in the Victorian 3D digital cadastre investigation

As shown in Figure 1, the first phase deals with developing geometrical validation rules. The second phase is related to the development of non-geometrical (semantic) validation rules and in the third phase, an online service similar to the current Victorian ePlan validation service ${ }^{5}$, will be implemented.

The aim of this paper is to discuss the progress of the first phase of 3D cadastral data validation research in Victoria. The four geometrical rules developed so far in this phase are discussed in detail. More validation rules will be developed during this research. This study has focused on LandXML data format, as the current digital data format in the Victorian digital cadastre project (ePlan). The rest of this paper is organised as follows: Section 2 gives an overview of the validation of 3D cadastral objects. Relevant studies are reviewed in Section 3. In Section 4, the development of four major 3D geometrical validation rules is addressed. Section 5 discusses the impacts of the proposed validation checks on the subdivision process and concludes with a direction for future research.

\section{The Need for Validating 3D Cadastral Objects}

Geometric correctness is a key factor for the quality of a 3D cadastre. Standards should define the measures and outcomes for geometric modelling of 3D objects. ISO (International Organisation for Standardisation) have developed standards for the data quality of spatial data. These standards establish the principles for describing the quality of geographic data and specify components for reporting quality

\footnotetext{
${ }^{5}$ Available at: https://www.spear.land.vic.gov.au/spear/eplanPublicServices/Prepare.do
} 
information. They also provide an approach to organising information about data quality (ISO 19113:2002) and provide procedures for determining and evaluating spatial data (ISO 19114:2003). In addition to ISO, OGC (Open Geospatial Consortium) have also developed OpenGIS Simple Features Specification for 2D data validation of spatial data (OpenGIS, 1999). For example, OGC define polygons as topologically closed objects.

Current standards do not precisely define a rigid base for data validation (Wagner et al., 2013). The data quality Working Group in OGC have defined some categories for quality measures, including accuracy, completeness, consistency and integrity, definition for semantic interoperability, language, projection, and scale (OGC, 2016). However, there is no accepted definition for the quality of 3D models. In addition, there is no standard for describing the best approach for geometric modelling in 3D cadastre. Based on the needs and availability of cadastral data, various methods should be considered. For example, ISO 19152:2012 - Land Administration Domain Model (LADM) supports five approaches for defining parcels. Therefore, the validation rules must be compatible with the method of geometric modelling.

The application domain also has an important impact on data modelling and validation. Different applications require adaptable product specifications which control the quality requirements. Therefore, specific 3D validation rules must be developed for cadastral purposes and the existing 3D validation rules might not be fully compatible with the business of cadastre. In addition, every jurisdiction has its own cadastral legislation and therefore; validation rules should be localised to be compatible with cadastral law. In the next section, literature of 3D data validation is reviewed.

\section{An Overview of Previous Relevant Studies}

Validation is a familiar topic in computing, generally as a mechanism to protect a database from the effects of inappropriate data (Karki et al., 2013). Some researchers have looked into 3D validation and developed some ideas and theories for validation of 3D cadastral objects. Thompson and Van Oosterom (2014) developed a rigorous axiomatic definition of a 3D parcel (spatial unit), and its relationship with neighbouring parcels. They also developed about 10 axioms using mathematical formalism to check various data scenarios for validating 3D cadastral data. Thompson et al. (2012) extended a set of axioms that can be used for formalising the validation of 3D cadastral data specifically to LADM. As an example, two nodes cannot be closer than the acceptable threshold.

Karki et al. (2010) discussed some object reconstruction approaches including tetrahedrons, objects from simpler solids, regular polytope, and extrusion. They also proposed some requirements for validating 3D cadastral data based on various possible scenarios. They classified the validation rules into the following groups:

- Internal validity of 3D parcels

- Surface or base parcel

- Relationships to other parcels

- Unique geometrical situations

- Further processing on the geometry

- Entry level validations

However, the validation requirements were only described in their paper and there is no information about the method of implementation. 
Wagner et al. (2013) described the validation of virtual urban models for CityGML format. They focused on common errors which are typically found in 3D city models. Alama et al. (2013) looked at geometric and semantic validation of CityGML data. They proposed a workflow for detecting geometrical and semantical errors in CityGML. These rules have been implemented as part of CityDoctor research project.

Topology has also been considered for implementing 3D validation. Gröger and Plümer (2009) developed a set of axioms to control the spatial consistency of 3D models. The axioms were developed in a modular method using topological surfaces. Zhao et al. (2012) proposed using topological relationships for validation in 3D cadastre. Among different possible scenarios in topological relations, touch and disjoint of 3D objects are acceptable and intersection is not acceptable. In their paper, they only focused on touch scenarios.

Kazar et al. (2008) looked at the problems of validating 3D geometries in Oracle database. They defined some validation rules such as the closedness test (the solid object must be closed), connectedness test, inner-outer check, and the orientation check to check the data quality. They discussed validation of 3D geometries and modelled various 3D geometries in Oracle and defined rules for 3D valid geometries. Oracle has also developed some validation checks in their Oracle Spatial database ${ }^{6}$.

Some software vendors have also developed 3D validation rules in their packages. For example, CityDoctor ${ }^{7}$ is a free tool for checking the quality of 3D city models. Various 3D validation rules have been developed in this software to control the accuracy of 3D models. For example, it checks duplicated points, self-intersection, face orientation, and co-planarity. Some limited validation checks have also been implemented in other applications such as Solibri Model Checker, Navisworks Manage, and Bentley Microstation.

Based on the literature, the previous research have mainly focused on providing a general mathematical definition of validation checks for 3D objects. Whereas, this paper aims to focus on the cadastre domain and develop mathematical approaches for validating 3D cadastral objects. In the next section, the development of four geometrical validation rules for the Victorian 3D digital cadastre is discussed.

\section{Development of Geometrical Validation Checks for 3D Cadastral Objects - The Victorian Case Study}

The current examination process for building subdivision plans requires various controls including both geometrical and semantical checks. Appendix 1 lists all Victorian 2D ePlan geometrical and semantical validation rules. The semantical rules will also apply to $3 \mathrm{D}$ ePlans through minimal modifications. However, because the nature of 2D and 3D ePlan modelling is different, there is a need to develop some specific geometrical rules for 3D ePlans. Accordingly, in this section some of the prevalent issues in the building subdivision plans are first presented and then, the four geometrical validation rules developed in the current research to address those issues are discussed in detail. According to Appendix 1 , to align $3 \mathrm{D}$ validation rules with the current $2 \mathrm{D}$ rules there is a need to develop some other geometrical rules in the next step of this research.

In the subdivision process, plans submitted by the land surveyor are checked for possible overlap or gap among parcels. Small overlaps or gaps cannot be easily identified, specially in PDF or paper based

\footnotetext{
${ }^{6} \mathrm{https}: / /$ docs.oracle.com/cd/E11882_01/appdev.112/e11830/sdo_intro.htm\#SPATL474

${ }^{7} \mathrm{http} / / / \mathrm{www} . c i t y d o c t o r . e u /$ index.php/citydoctor_for_3d_city_models.html?language=en
} 
plans. However, in ePlan files, this error is one of the common errors and can be easily identified. Figure 2 shows a gap between lot 1 and 2 in a $2 \mathrm{D}$ subdivision plan.

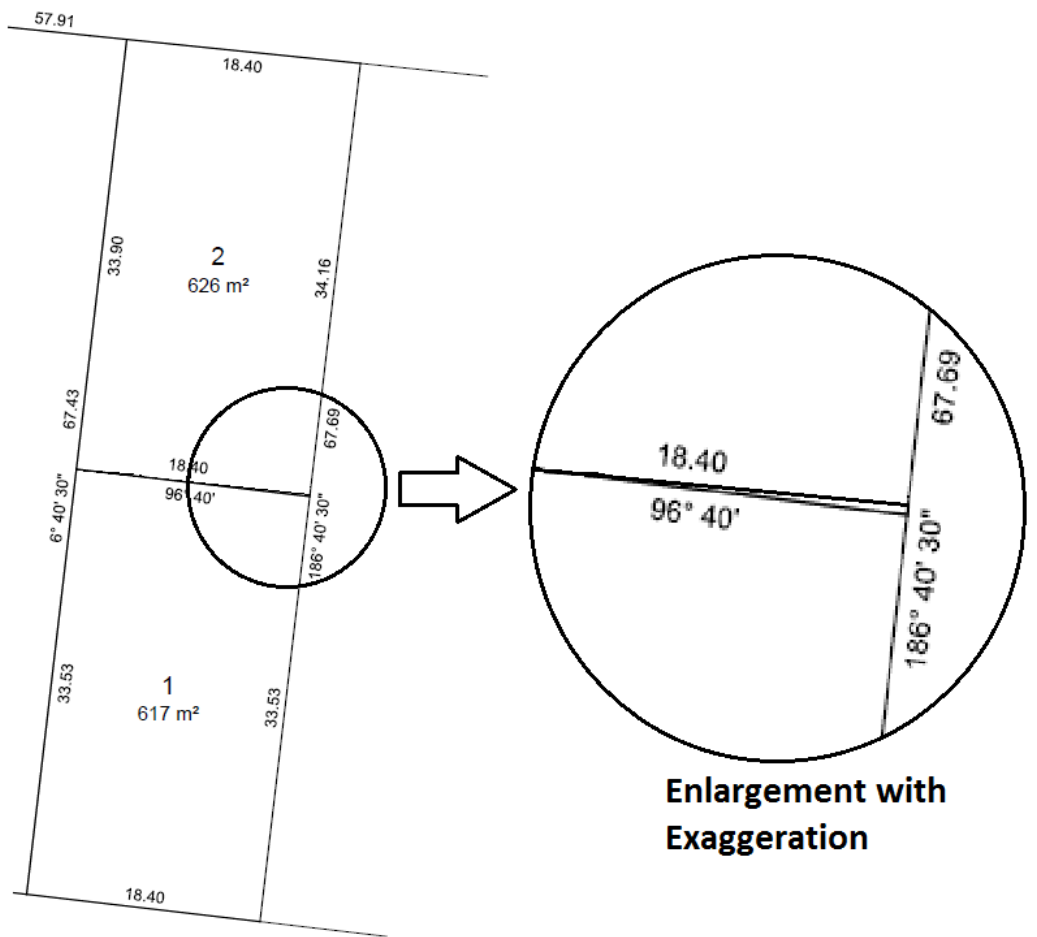

Figure 2. A gap between parcels in a subdivision plan

This error usually happens when boundaries do not match properly. In a 3D case, this data issue brings a gap or a clash between parcels. Validation rules one and two, discussed below, look at these issues.

Non-flat faces also bring gaps to 3D objects. Therefore, there is a need for a validation rule to ensure that all faces are flat to avoid any gap in 3D objects. In LandXML data model, elements can be defined as planar or non-planar faces. However, for a given boundary, there are many possible permutations when complicated objects are created with non-planar faces. For example, in Figure 3, two different scenarios are possible in the rendering process of a non-planar face on the top. These two scenarios might give different answers when the volume is calculated. In addition, an encroachment into the region of ambiguity could cause an un-resolvable dispute. To avoid this undefined behaviour, nonplanar faces can be triangulated to match what is expected. In general, planar faces on polygon meshes are preferred. Non-planar faces may render incorrectly in the final image or when exported to an interactive visualisation application (Autodesk Maya, 2014). Validation rule three, discussed below, examines this problem.

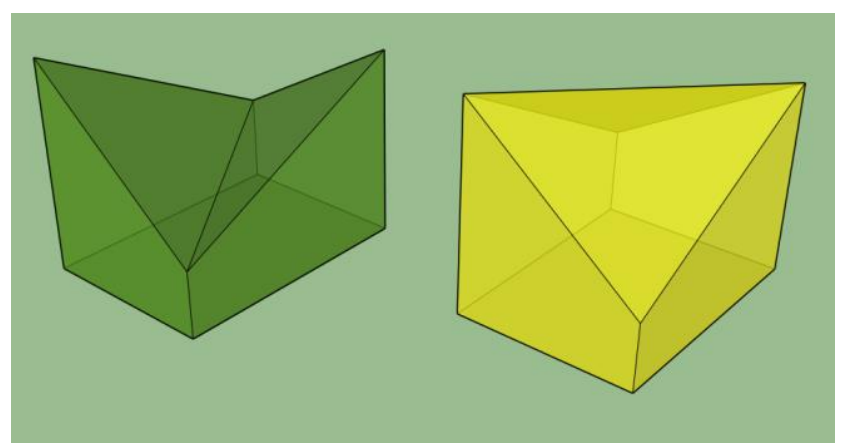


Figure 3. Two possible scenarios of creating a 3D object with a non-planar face (Shojaei et al., 2016)

The other common issue is parcel misclosure. According to the Victorian regulations, 2D parcels must be closed with an acceptable tolerance. The length of the misclosure vector should not exceed 15 millimetres +100 parts per million of the perimeter (Victorian Consolidated Regulations, 2015). If the length of misclosure does not exceed the tolerance, the error is acceptable and observations must be adjusted. This error is likely to happen in 3D model creation as well. In this case, some faces do not completely match and gaps will be created in 3D parcels. If there is no gap in a 3D object, this object is considered watertight which refers to a 3D object that can hold water without any holes, cracks or missing faces (Karki et al., 2010). Validation rule four, discussed below, examines this problem.

The following rules include six sections. The 'Equivalent 2D ePlan Validation Rule' section describes how this rule is linked to the existing validation rules for 2D ePlans. The 'Definition' and 'Description' sections explain the rule and its importance. The 'Method' and 'Condition' sections address the methodology of implementation and the exceptions/special requirements respectively. The 'Cadastral Requirement' section addresses the relationship between a validation rule and the Victorian cadastre requirements.

\subsection{Validation Rule 1 - Created Parcels for Cancelled Parcels}

- Equivalent 2D ePlan Validation Rule

VR072 - Title Boundary Consistency - The outer boundary of created primary parcels must be coincident with the outer boundary of extinguished parcels.

- Definition

In a plan of subdivision, created parcels (e.g. lots and common property) must occupy the whole space of the extinguished (cancelled) parcel(s) with an acceptable error in volume calculation.

- Description

In a full 3D cadastre, the 3D space is subdivided into volumes partitioning the 3D space without overlaps or gaps (Stoter and Salzmann, 2003). Therefore, all spaces are partitioned and each partition must have at least one owner. This is called partitioning of space. Following this concept, there should be a consistency between the volume of created parcels and the extinguished one(s). For example, if one parcel is subdivided into two lots, the sum of the volume contained in each new lots must be the same as the volume of the extinguished parcel with consideration given to acceptable errors in volume calculation. This error can be defined as an acceptable threshold (Figure 4).

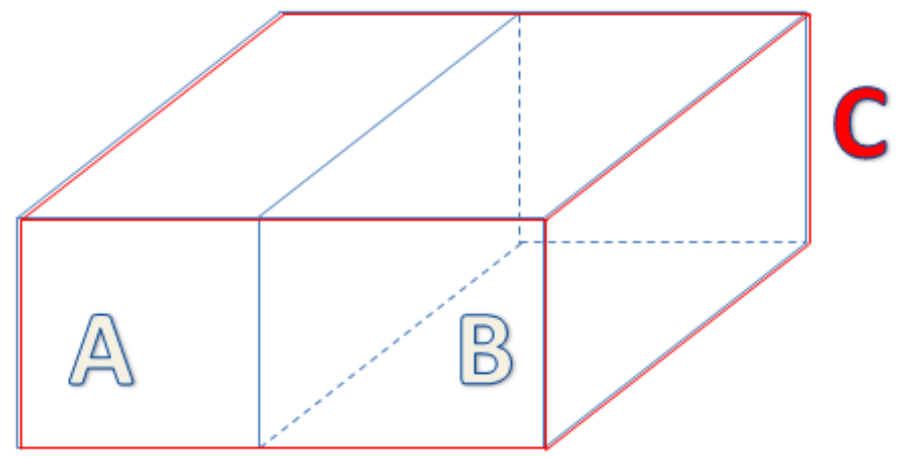


Figure 4. Given A, B and C volumetric objects, A \& B represent new created volumes based on the extinguished one $(\mathrm{C})$

\section{- Method}

In order to check the volumetric consistency, the volume of the parcels must be computed. There are many approaches for calculating the volume of 3D objects. Cadastral objects are not always like Figure 4 as they may be very complex, including curved surfaces. To calculate the volume of 3D objects, a method by Zhang and Chen (2002) was considered.

In a LandXML file, 3D objects are represented by a set of vertices and faces. Before volume calculation, some pre-processing on 3D objects is required. Firstly, all polygons must be triangulated which is commonly used in mesh coding, mesh signal processing, and mesh editing. Then, the normal vector of each triangle must to be calculated. This can be determined by the order of the vertices and the righthand rule, as shown in Figure 5.

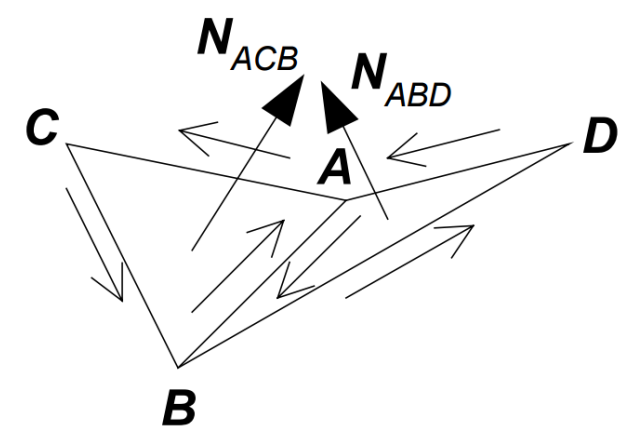

Figure 5. Normal vectors and order of vertices (Zhang and Chen, 2002)

The consistency direction of normal vectors for two neighbouring triangles is checked by controlling the directions of the shared edges. For example, in Figure 5, $\mathrm{AB}$ is the common edge of triangle ACB and $\mathrm{ABD}$. In this figure, the direction is from $\mathrm{B}$ to $\mathrm{A}$, and in triangle $\mathrm{ABD}$, the direction is from $\mathrm{A}$ to $\mathrm{B}$, thus $\mathrm{N}_{\mathrm{ACB}}$ and $\mathrm{N}_{\mathrm{ABD}}$ are consistent.

The volume calculation is based on elementary calculation of tetrahedrons. For each triangle, we connect each of its vertices to the origin $(0,0,0)$ and form a tetrahedron, as shown in Figure 6.

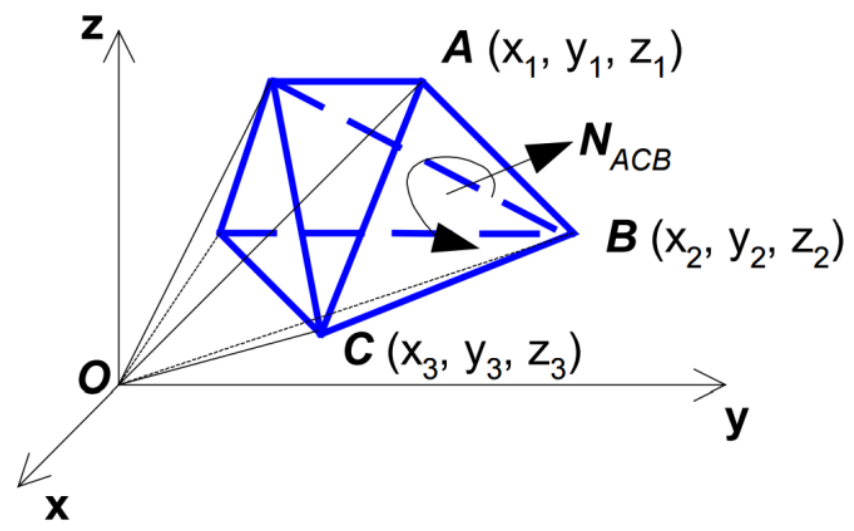

Figure 6. Volume calculation method for 3D objects (Zhang and Chen, 2002) 
Then, the volume of each tetrahedron is calculated. The sign (positive or negative) of each volume is determined with respect to the direction of the normal vector.

In Figure 6, triangle $\mathrm{ACB}$ has a normal $\mathrm{N}_{\mathrm{ACB}}$ and the volume of $\mathrm{OACB}$ is calculated by:

$$
\left|V_{O A C B}\right|=\left|\frac{1}{6}\left(-x_{3} y_{2} z_{1}+x_{2} y_{3} z_{1}+x_{3} y_{1} z_{2}-x_{1} y_{3} z_{2}-x_{2} y_{1} z_{3}-x_{1} y_{2} z_{3}\right)\right|
$$

As the origin $\mathrm{O}$ is at the opposite side of $\mathrm{N}_{\mathrm{ACB}}$, the sign of this tetrahedron is positive. The sign can also be calculated by inner product $\overrightarrow{\mathrm{OA}} \cdot \mathrm{N}_{\mathrm{ACB}}$. Therefore, the total volume is the sum of each volume calculated based on:

$$
\begin{gathered}
V_{i}^{\prime}=\frac{1}{6}\left(-x_{i 3} y_{i 2} z_{i 1}+x_{i 2} y_{i 3} z_{i 1}+x_{i 3} y_{i 1} z_{i 2}-x_{i 1} y_{i 3} z_{i 2}-x_{i 2} y_{i 1} z_{i 3}-x_{i 1} y_{i 2} z_{i 3}\right) \\
V_{\text {total }}^{\prime}=\sum_{i} V_{i}^{\prime}
\end{gathered}
$$

In these equations, $i$ stands for the index of triangles $\left(x_{i 1}, y_{i 1}, z_{i 1}\right),\left(x_{i 2}, y_{i 2}, z_{i 2}\right)$ and $\left(x_{i 3}, y_{i 3}, z_{i 3}\right)$, which are coordinates of the vertices of triangle $i$. The volume of a 3D object is always achieved by the absolute value of $\mathrm{V}_{\text {total }}^{\prime}$.

Subsequently, the volume of created primary parcels must be equal to the volume of the extinguished parcel by considering an acceptable threshold.

- Conditions

Due to expected random errors in surveying observations, an acceptable error should be considered in volume calculation.

- Cadastral requirement

This validation helps to avoid creating and/or losing 3D spaces at the time of subdivision or consolidation of $3 \mathrm{D}$ spaces. This is one of the common errors in 2D subdivision and consolidation plans.

\subsection{Validation Rule 2 - Parcel Collision (Clash)}

- Equivalent 2D ePlan Validation Rule

VR117 - Overlapping Parcels - Created primary parcels (Lot, Stage Lot, Road, Reserve, Common Property, Crown Allotment, Crown Portion) must not overlap each other.

- Definition

In 3D cadastre, ownership spaces cannot intersect. It means a space cannot be occupied by more than one object. Therefore, a validation check is required to avoid intersecting parcels (Figure 7). 


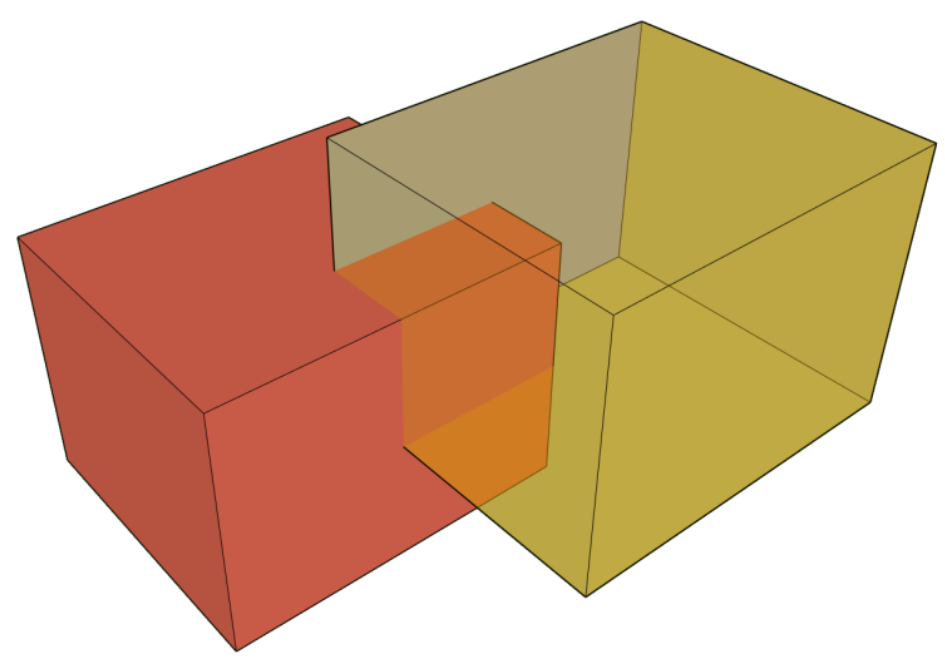

Figure 7. Collision between two 3D objects

\section{- Description}

There are several methods for detecting collision of 3D objects. Some of them are quick, but not accurate (e.g. ray tracing) and some are slow in running the test but more accurate in the results. In fact, faster algorithms can be achieved by a bounding box or bounding sphere to every pair of polyhedra, or by octree or voxel methods for even better speed performance (Moore and Wilhelms, 1988).

Due to the importance of collision detection in 3D cadastre, an accurate algorithm developed by Moller (1997) was considered. This algorithm is based on triangle-triangle intersection which is useful for mesh collision detection. Triangle-triangle collision can use the Separating Axis Theorem to detect the collision.

In this method, the 3D model must be triangulated. Then, all triangles are checked and intersections are detected. For intersections, the three vertices of one triangle are tested against the plane of the other triangle. If all the vertices of the first triangle are on the same side of the plane, the two triangles are separated and there is no collision. However, if vertices are on both sides of the plane, the similar test is done for the other triangle. If vertices are on both sides of the other plane, there is an intersection. Then, these intersection lines are calculated. If there is an overlap for these two lines, the triangles have collision (Figure 8).
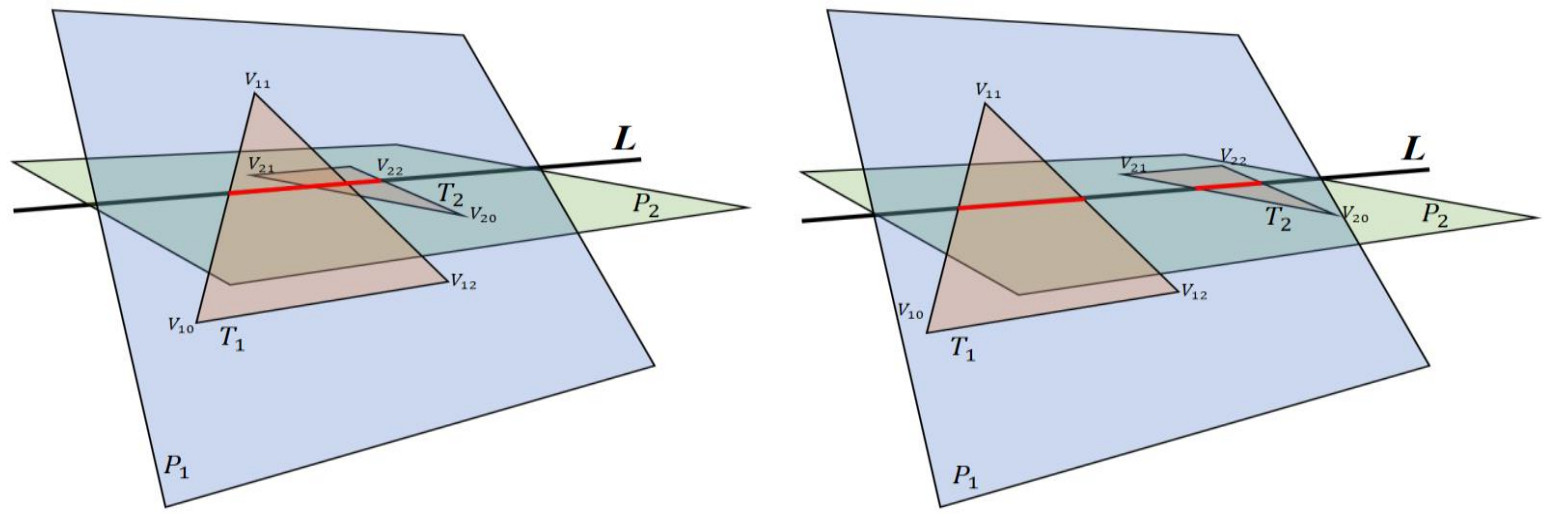
Figure 8. Triangles with intersection on left, and triangles without intersection on the right. The intersection line is calculated (marked red in both figures) and any overlaps are determined. There is no overlapping on the right; therefore, there is no collision. Adapted from (Moller, 1997)

- Method

There are two triangles as $\mathrm{T}_{1}$ and $\mathrm{T}_{2}$ and the vertices of these triangles are $V_{10}, V_{11}, V_{12}$ and $V_{20}, V_{21}, V_{22}$ respectively. The planes made from triangles are called $\mathrm{P}_{1}$ and $\mathrm{P}_{2}$.

The first plane equation is $P_{1}: N_{1} . X+d_{1}=0$ which $\mathrm{X}$ is any point on the plane.

$$
\begin{gathered}
N_{1}=\left(V_{11}-V_{10}\right) \times\left(V_{12}-V_{10}\right) \\
d_{1}=-N_{1} . V_{10}
\end{gathered}
$$

Then, the signs (positive or negative) and distances of $T_{2}$ vertices to $P_{1}$ is calculated by inserting the vertices into the plane equation:

$$
d_{V_{i}^{1}}=N_{1} \cdot V_{2 i}+d_{1}, i=0,1,2 .
$$

If all $d_{V_{i}} \neq 0, i=0,1$ and 2 , it means no point is on the plane and if distances have the same sign, then $T_{2}$ is located on one side of $P_{1}$ and there is no overlap. A similar test is done for $T_{1}$ and $P_{2}$. These tests optimise the process speed by avoiding a lot of computations for many triangles.

If all $d_{V_{i}^{1}}=0, i=0,1$ and 2 , it means two triangles are coplanar.

If all $d_{V_{i}^{1}} \neq 0, i=0,1$ and 2 , and the distances have different sign, it means $\mathrm{T}_{2}$ and $\mathrm{P}_{1}$ intersects and the intersection of $\mathrm{T}_{2}$ and $\mathrm{P}_{1}$ is a line (Line $L$ in Figure 8), $L=O+t D$, Where $D=N_{1} \times N_{2}$ is the direction of intersection line and $\mathrm{O}$ is points on this line. There would be the same test for $\mathrm{T}_{1}$ and $\mathrm{P}_{2}$. These intersections create intervals on L. If these intervals overlap, it means the triangles overlap and therefore, these triangles have collision.

\section{- Conditions}

The rule is applicable to triangulated meshes. However, this method can be easily applied to nontriangulated meshes with only flat faces.

- Cadastral requirement

A clash in 3D cadastre is usually considered as an important error and must be detected. However, there are some exceptions as some clashes are acceptable. For example, secondary interests such as easements, restrictions and depth limitations float over base cadastral parcels as these are not defined as a created primary parcel (eg. Lot). Therefore, they can intersect any base parcels or other secondary interests. Figure 9 shows an easement within a parcel. 


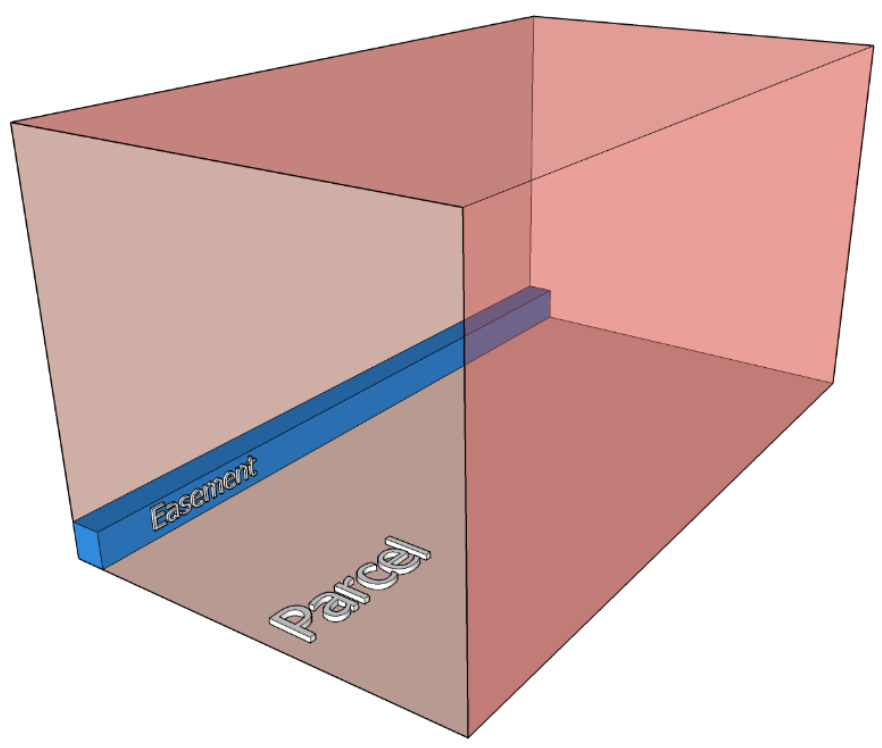

Figure 9. An easement is intersecting a parcel and it is an exception in having an acceptable clash.

\subsection{Validation Rule 3 - Flat Faces}

- Equivalent 2D ePlan Validation Rule

Not Applicable to 2D ePlans.

\section{- Definition}

In the boundary representation approach for geometric modelling, all faces that make the 3D object must be flat.

\section{- Description}

In LandXML, the boundary representation approach is used for modelling 3D objects (Shojaei et al., 2016). In boundary representation, all faces must be flat. Therefore, all vertices in a face should be in the same plane.

\section{- Method}

Various methods exist to check the flatness of all faces. If a face is made of three separate points, it makes a flat face and if a face is composed of more than three separate points, all of them must be in the same plane to be planar. Otherwise, these create a 3D object (e.g. a parallelepiped from four nonplanar points). To check whether these points are planar or not, the volume of the created parallelepiped should be calculated. If the volume is zero, it means the four points are coplanar. Otherwise, the face is not flat. Based on Figure 10, volume of a parallelepiped made from 4 points $(\mathrm{P}, \mathrm{Q}, \mathrm{R}$, and $\mathrm{S})$ is calculated by the following equation (Nykamp, 2017):

$$
\begin{aligned}
\text { Volume }= & \|a \times b\|\|c\||\cos \emptyset|=|(a \times b) \cdot c| \\
& (a \times b) \cdot c=\left|\begin{array}{lll}
c_{1} & c_{2} & c_{3} \\
a_{1} & a_{2} & a_{3} \\
b_{1} & b_{2} & b_{3}
\end{array}\right|
\end{aligned}
$$




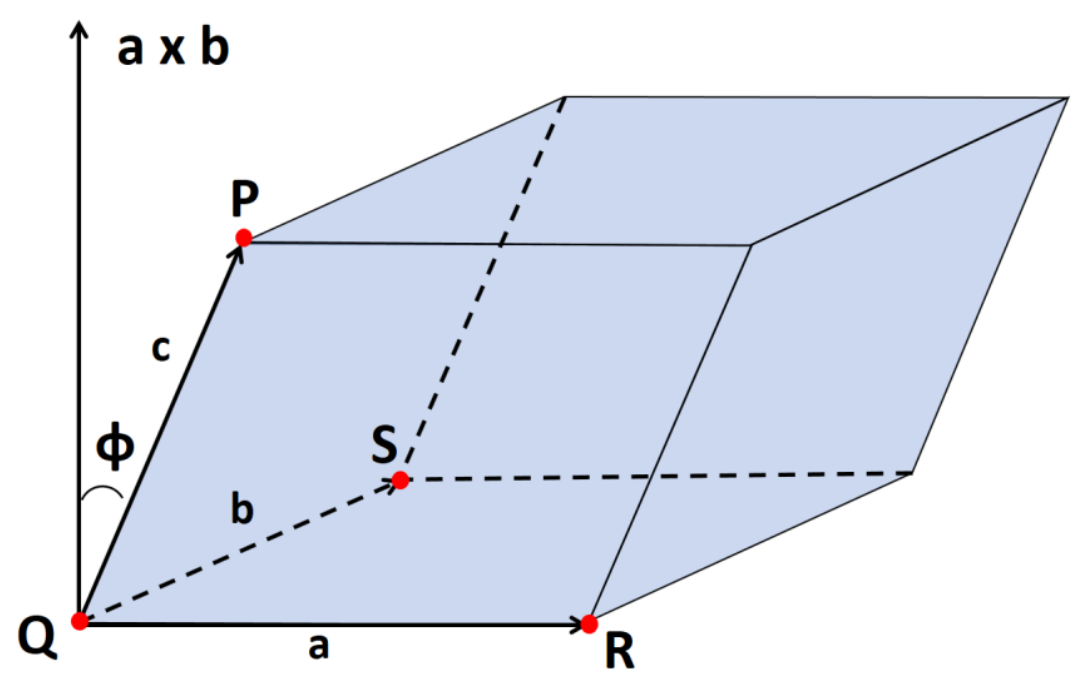

Figure 10. A volume calculation method for a parallelepiped (Nykamp, 2017)

In this approach, if a face is composed of only four separated points $(\mathrm{P}, \mathrm{Q}, \mathrm{R}$, and $\mathrm{S})$, these points are considered for volume calculation. If a face is composed of more than four separate points, each set of four points is considered for this calculation.

- Conditions

A threshold should also be considered to avoid random errors in measurements.

- Cadastral requirement

A non-flat face might bring gaps to the created 3D objects when they are modelled. This results in a non-acceptable 3D object in LandXML data format. Therefore, this validation rule is required to confirm the correctness of each face to make correct 3D objects.

\subsection{Validation Rule 4 - Parcel Geometry Closure}

- Equivalent 2D ePlan Validation Rule

VR071 - Parcel Observations Closure - All parcel geometries are expected to "close" within the allowable tolerances defined in Regulation 7 of Surveying (Cadastral Surveys) Regulations 2015.

- Definition

This rule is required to avoid any misclosure in creating 3D objects. 3D objects are expected to be "closed".

- Description

The closure of a parcel geometry can be checked through a consistency check using Euler's formula (Ericson, 2005). It is assumed that each edge is shared by exactly two faces, that each edge is connected to exactly two vertices, and that at least three edges join at each vertex.

- Method 
The mathematical relationship between the number of vertices $(V)$, faces $(F)$, and edges $(E)$ of a simple polyhedron is stated in Euler's formula:

$$
V+F-E=2
$$

For example, the polyhedron in Figure 11 (a) meets the formula because there are nine vertices. Also, nine faces make the object and there are 16 edges which satisfies the above formula $(9+9-16=2)$.

For a non-simple polyhedron the above equation does not satisfy the formula (Figure 11(b)). Nonsimple Polyhedrons have one or more holes in them. The number of holes is referred to as the genus of the polyhedron. An extended version of the aforementioned equation is Euler-Poincare formula which considers a polyhedron with genus $(\mathrm{G})$.

(a)

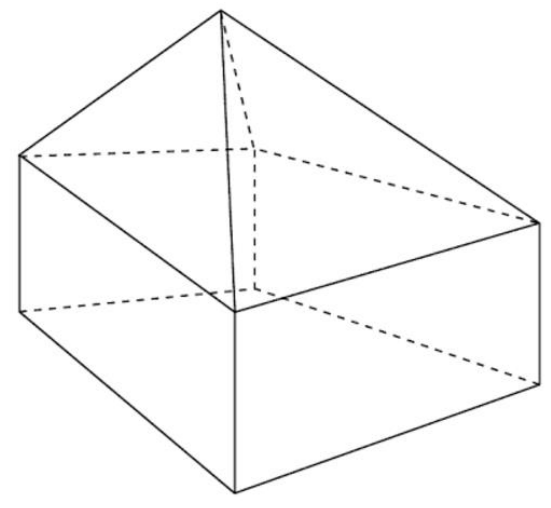

Figure 11. (a) A simple polyhedron, (b) A non-simple polyhedron (of genus one) (Ericson, 2005)

For a non-simple polyhedron the following equation applies:

$$
V+F-E=2(1-G)
$$

For example, in Figure 11(b), $V=16, F=16, E=32$, and $\mathrm{G}=1$. Thus, 16+16-32=2(1-1) which satisfies the above-mentioned formula. According to Ericson (2005) both formulas apply to manifold geometry.

Ericson (2005) states that if a closed mesh consists only of triangles, the number of edges relate to the number of triangles $(\mathrm{T})$ as $2 \mathrm{E}=3 \mathrm{~T}$ and the corresponding formulas become $\mathrm{T}=2 \mathrm{~V}-4$ and $\mathrm{E}=$ $3 \mathrm{~V}-6$. Regarding a mesh made by quads, the edges would relate to the number of quads $(\mathrm{Q})$. The relationships between the number of edges, vertices and faces of a closed mesh are detailed in Table 1.

Table 1. Euler's derived formula for a closed mesh (Ericson, 2005)

\begin{tabular}{|l|l|}
\hline $\begin{array}{l}\text { For a closed (manifold) mesh consisting } \\
\text { of a number of... }\end{array}$ & $\begin{array}{l}\text { The number of edges }(\mathbf{E}), \text { vertices }(\mathbf{V}) \text { and } \\
\text { faces relates as... }\end{array}$ \\
\hline Triangles $(T)$ & $2 E=3 T, T=2 V-4, E=3 V-6$ \\
\hline Quads $(Q)$ & $2 E=4 Q, Q=V-2, E=2 V-4$ \\
\hline Triangles $(T)$ and Quads $(Q)$ & $2 E=3 T+4 Q, T=2 V-2 Q-4$ \\
\hline
\end{tabular}


An example of the formula's application can be applied in Figure 12. Both cubes consist of two triangles and six quads. When computing the number of edges $E=(3 T+4 Q) / 2$, the $12(a)$ cube has (15) but the 12(b) cube has (14.5). According to Ericson (2005), because the latter result is not an integer number, cube 12(b) is not correctly formed. As a matter of fact, the 12(b) cube has a T-junction on the top face (a crack shown in grey).

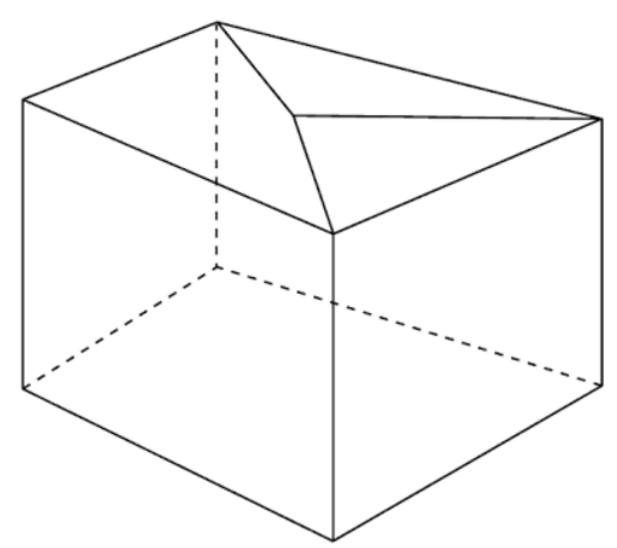

(a)

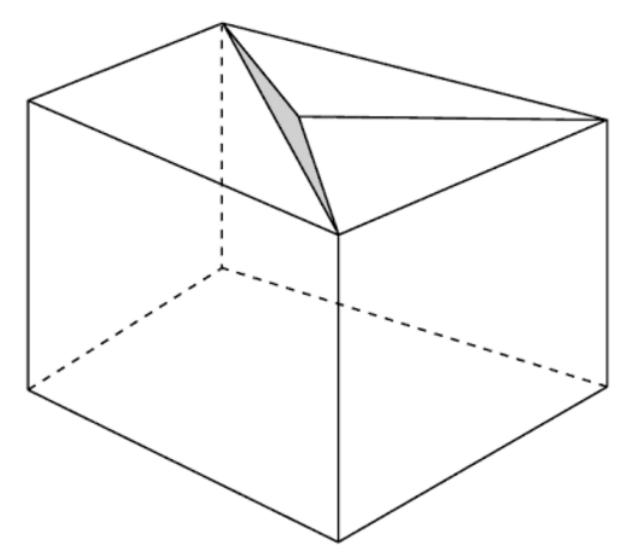

(b)

Figure 12. Closed manifold meshes consisting of quads and triangles (Ericson, 2005)

- Conditions

The aforementioned formulas are useful but may not validate a malformed mesh. Thus, the validator must carefully check the geometry of the mesh before applying any formula.

- Cadastral requirement

According to the Surveying Regulations 2015 in Victoria (Victorian Consolidated Regulations, 2015), a 2D parcel must be closed. Similarly, 3D objects must be watertight to avoid different types of geometrical and topological errors in 3D object definition.

\section{Discussion and Conclusion}

The State of Victoria in Australia has been moving towards a digital cadastre since the introduction of ePlan in this jurisdiction in 2011. ePlan is a collaborative program between the Australian land authorities and the surveying industry, in conjunction with ICSM which aims to replace paper and PDF plans with digital files. ePlan has been implemented for 2D cadastral plans in Victoria; however, the support of 3D cadastral plans in ePlan is still under investigation. The Victorian 3D digital cadastre investigation consists of the technical, legal and institutional aspects. The technical aspects of this investigation, which includes 3D data modelling, storage, validation, and visualisation has been studied since 2014.

Following a review of the current status of 3D cadastre validation around the world, this paper has discussed the latest outcomes of 3D cadastral data validation research in Victoria. This research has 
classified the 3D data validation into two categories: a) geometrical and b) non-geometrical (semantical) rules.

Currently, there are 128 validation rules including 12 geometrical rules and 116 semantical rules for checking the quality of 2D ePlans in Victoria (see Appendix 1). The existing 2D ePlan validation service has been developed as a web service which is connected to several databases for checking the content of an ePlan file, such as survey marks, road names, parcel address and title details. This significantly facilitates the examination process as the plan examiners look at the results of the report, eliminating the need to view multiple databases for consistency checking.

The ePlan validation service supporting both $2 \mathrm{D}$ and 3D ePlans will provide significant efficiency gains to the ePlan examination process. Three of the main benefits of this service are listed below:

- Pre-lodgement check: The ePlan validation service enables the surveyors to undertake a prelodgement check on their ePlans to ensure the completeness and correctness of the ePlan content. The validation service potentially replaces the quality assurance (QA) process undertaken by surveying firms when preparing PDF plans. The validation service can be run in two environments: a) ePlan services web page, b) surveying software packages (e.g. AutoCAD Civil 3D Stringer ePlan). The latter speeds up the validation process since the validation report is generated within the software package through a web service.

- Quicker plan examination and registration time frames: plan examiners can rely on the validation report as part of their plan examination process. Reviewing the validation report will enable plan examiners to readily identify potential problems in the ePlan data. Without this report, they need to manually examine plans which is very time consuming and error prone, particularly for complex or multi-lot subdivision plans.

- Less requisitions: At LUV, approximately $50 \%$ of plans are requestioned by examiners due to the data issues in plans. This results in significant delays in the registration process, which affects all stakeholders, such as surveyors, architects, developers, councils, and referral authorities. This delay also imposes significant holding costs to the land development process, as the properties cannot be released to the market until registration of the plan. Using the ePlan validation service, the ePlans can be checked for the most common issues before getting to the examination stage. This will significantly reduce the plan requisitions.

To achieve a comprehensive validation service that can cover both 2D and 3D ePlan, an investigation was defined in three phases, as discussed in Section 1. In the first phase of this investigation, a subset of existing 12 geometrical rules, which considers the most common issues in the subdivision plan examination process, was selected. The selected rules were further developed to support the 3D ePlans. The outcomes of this research have been presented in the form of four geometrical validation rules.

The first validation rule looks at the issues regarding subdivision of parcels. The applied method is based on volume calculation. However, if there is any clash between 3D objects in complex scenarios, this validation rule might not be able to detect them. Consequently, the second validation rule has been developed to support the first rule as a pre-condition, to make sure that there is no clash detected between 3D objects for volume calculation. Validation rule two is comprehensive to detect clashes in complex 3D objects, as it detects clashes based on the triangulation technique. Therefore, all 3D objects will be triangulated and tested by this validation rule. The third validation rule looks at individual faces and detects non-flat faces in 3D models. Validation rule four checks the geometry closure. The combination of validation rule 3 and 4 assures 3D objects are correctly modelled and there is no topological issue in the data. Once the other required geometrical validation rules are developed in 
phase one, various test cases will be developed to test the effectiveness of these rules in simple and complex scenarios.

To complete the first phase of the current investigation, the Victorian ePlan team will study how the remaining 2D geometrical rules can be extended to support 3D ePlans. In the second phase, the semantical validation rules listed in Appendix 1 will be studied to determine whether they can support the 3D ePlans as they currently are, or modification is required. In phase three, the current validation web service will be developed to support the geometrical and semantical validation rules for 3D ePlans.

\section{References}

1. Aien, A., Kalantari, M., Rajabifard, A., Williamson, I., Shojaei, D. (2012). Developing and testing a 3D cadastral data model: a case study in Australia. ISPRS Annals of the Photogrammetry, Remote Sensing and Spatial Information Sciences, Volume I-4, 2012 XXII ISPRS Congress, 25 August - 01 September 2012, Page 1-6, Melbourne, Australia

2. Aien A., Rajabifard A., Kalantari M., Williamson I., Shojaei D. (2014). Development of XML Schemas for Implementation of a 3D Cadastral Data Model, 4th International Workshop on 3D Cadastres 9-11 November 2014, Page 131-158, Dubai, United Arab Emirates

3. Aien, A., Rajabifard, A., Kalantari, M., Shojaei, D. (2015). Integrating Legal and Physical Dimensions of Urban Environments, ISPRS International Journal of Geo-Information, 2015, 4(3), 1442-1479; doi:10.3390/ijgi4031442

4. Alama, N., Wagner, D., Wewetzer, M., von Falkenhausen, J., Coors, V., Pries, M. (2013). Towards Automatic Validation And Healing Of Citygml Models For Geometric And Semantic Consistency, ISPRS Annals of the Photogrammetry, Remote Sensing and Spatial Information Sciences, Volume II2/W1, ISPRS 8th 3DGeoInfo Conference \& WG II/2 Workshop, 27 - 29 November 2013, Istanbul, Turkey

5. Arens, C., Stoter, J., van Oosterom, P. (2005). Modelling 3D spatial objects in a geo-DBMS using a 3D primitive, in Computers \& Geosciences Journal, 31(2): 165-177

6. Ericson, C. (2005). Real-Time Collision Detection. San Francisco: Morgan Kaufmann Publishers, CRC Press, Taylor \& Francis Group

7. Gerig, G., Jomier, M., Chakos, M. (2001). Valmet: A New Validation Tool for Assessing and Improving 3D Object Segmentation. http://link.springer.com/chapter/10.1007/3-540-45468-3_62

8. Gröger G, Plümer L. (2009). How to achieve consistency for 3D city models. GeoInformatica 15(1):137-165

9. Ho, S., Rajabifard, A., Kalantari, M. (2015). 'Invisible' constraints on 3D innovation in land administration: A case study on the city of Melbourne, Land Use Policy, Volume 42, January 2015: $412-425$

10. ICSM (2015). Cadastre 2034 Strategy, Powering Land and Property, http://www.icsm.gov.au/cadastral/Cadastre2034.pdf

11. International Organization for Standardization (ISO) (2002). ISO 19113 standard: geographic information - quality principles. Available at: http://www.iso.org/iso/iso_catalogue/catalogue_tc/catalogue_detail.htm?csnumber=26018 
12. International Organization for Standardization (ISO) (2003). ISO 19114 standard: geographic information — quality evaluation procedures. Available at:

http://www.iso.org/iso/iso_catalogue/catalogue_tc/catalogue_detail.htm?csnumber=26019

13. International Organization for Standardization (ISO) (2012). ISO 19152 Geographic information -Land Administration Domain Model (LADM). Available at:

http://www.iso.org/iso/catalogue_detail.htm?csnumber=51206

14. Karki, S., Thompson, R., McDougall, K. (2010). Data validation in 3D cadastre. En T. Neutens, P. Maeyer, Developments in 3D Geo-Information Sciences, 92-122. Springer Berlin Heidelberg

15. Karki, S., Thompson, R., McDougall, K. (2013). Development of validation rules to support digital lodgement of 3D cadastral plans. In Computers, Environment and Urban Systems, 34-45

16. Kazar, B., Kothuri, R., van Oosterom, P., Ravada, S. (2008). On Valid and Invalid Three-Dimensional Geometries. In: Advances in 3D Geoinformation Systems. Van Oosterom P, Penninga F, Zlatanova S, Fendel E (Eds). Springer, Berlin.

17. Moore, M., Wilhelms, J. (1988). Collision Detection and Response for Computer Animation. In Computer Graphics (SIGGRAPH 1988 Proceedings), Volume 22, Number 4. ACM Press

18. Moller, T. (1997). A fast triangle-triangle intersection test. J. Graph. Tools, 2(2):25-30

19. Nykamp, D. (2017). The scalar triple product. From Math Insight. accessed 30 January 2017, http://mathinsight.org/contributor/dqnykamp

20. Olfat, H., Shojaei, D., Briffa, M. (2016). Victorian Digital Cadastre- Challenges and Investigations, In Proceedings of the 3rd Annual Conference of Research@Locate, Melbourne, Australia, 47-52

21. Olfat, H., Shojaei, D., Briffa, M., and Rajabifard, A. (2017). The Current Status and Ongoing Investigations of 2D and 3D Digital Cadastre (ePlan) in Victoria, Australia, 10th International Symposium on Digital Earth \& Locate17, 3-6 April 2017, Sydney, Australia

22. OpenGIS (1999). Simple Features Specification for SQL, Revision 1.1, https://portal.opengeospatial.org/files/?artifact_id=829

23. OGC (2016). Data Quality DWG, http://www.opengeospatial.org/projects/groups/dqdwg

24. Shojaei, D., Kalantari, M., Bishop, I., Rajabifard, A., Aien, A. (2013). Visualization Requirements for 3D Cadastral Systems. In Computers, Environment and Urban Systems, 41: 39-54

25. Shojaei, D. (2014). 3D cadastral visualisation: understanding users' requirements, PhD Thesis, The University of Melbourne.

26. Shojaei, D., Olfat, H., Rajabifard, A., Darvill, A., Briffa, M. (2016). Assessment of the Australian digital cadastre protocol (ePlan) in terms of supporting 3D building subdivisions, Land Use Policy Journal, 56: 112-124

27. Stoter, J., Salzmann, M. (2003). Towards a 3D cadastre: where do cadastral needs and technical possibilities meet? Computers, Environment and Urban Systems, 27: 395-410

28. Thompson, R., van Oosterom, P. (2012). Validity of Mixed 2D and 3D Cadastral Parcels in the Land Administration Domain Model, The Netherlands, 3rd International Workshop on 3D Cadastres: Developments and Practices 25-26 October 2012, Shenzhen, China

29. Thompson, R., van Oosterom, P. (2014). Axiomatic Definition of Valid 3D Parcels, Potentially in a Space Partition, 2nd International Workshop on 3D Cadastres, Delft, the Netherlands 
30. Victorian Consolidated Regulations (2015). Surveying (Cadastral Surveys) Regulations 2015, http://www.legislation.vic.gov.au/Domino/Web_Notes/LDMS/PubStatbook.nsf/b05145073fa2a882ca2 56da4001bc4e7/77EEB116E30CE6F8CA257E5800204AD2, Accessed 6 March 2017

31. Wagner, D., Wewetzer, M., Bogdahn, J., Alam, N., Pries, M., Coors, B. (2013). Geometric-semantical consistency validation of CityGML models. In J. Pouliot et al., eds. Progress and New Trends in 3D Geoinformation Sciences. Lecture Notes in Geoinformation and Cartography. 3D Geoinfo. Quebec, Canada: Springer Berlin Heidelberg

32. Zhang, C., Chen, T. (2002). Efficient Feature Extraction For 2D/3D Objects in Mesh Representation, International Conference on Image Processing, 3: 935-938

33. Zhao, Z., Guo, R., Li, L., Ying, S. (2012). Topological Relationship Identification in 3D Cadastre, 2012, 3rd International Workshop on 3D Cadastres, 2012, Shenzhen, China 


\section{D ePlan Validation Rule}

\section{Semantical Geometrical}

VR001 - ePlan Cadastral Information File Schema Validation Rule

Rule

VR002 - Survey Header Completenes

VR003 - Multipart Parcel Completeness

VR004 - Parcel Geometry Exists

VR005 - Easement Purpose Exists

VR006 - Owners Corporation Limitation Exists

VR007 - Admin Area Parcel Description Exists

VR008 - Road Parcel Description Exists

VR009 - Primary Parcel Address Exists

VR010 - Permanent Mark and Primary Cadastral Mark Completeness

VR011 - Reference Mark Completeness

VR012 - Instrument Point Completeness

VR014 - Instrument Setup to Point Reference

VR015 - Instrument Setup in Observation

VR016 - Unconnected Points

VR017 - Redundant Observations

VR018 - Annotation Parcel References

VR019 - Title Reference

VR020 - Date of Survey Plan Manual Check

VR021 - Plan Number Format

VR022 - Plan Number Allocation

VR023 - Surveyor Registration Number

VR024 - Existing Parcel in Victorian Online Title System (VOTS)

VR025 - Primary and Secondary Purpose Combination

VR026 - Purpose of Survey Section 22

VR027 - Purpose of Survey Section 23

VR028 - Purpose of Survey Section 24A

VR029 - Purpose of Survey Section 32

VR030 - Purpose of Survey Section 35 Vesting Manual Check

VR031 - Purpose of Survey Section 35, 32, 37 Manual Check

VR032 - Purpose of Survey Section 37 and 37(8)

VR033 - Planning Permit Annotation Manual Check

VR034 - Depth Limitation Manual Check

VR035 - LGA and Parish Exist

VR036 - Admin Area in Vicmap Admin

VR037 - Administrative Area Allocation

VR038 - Redundant Admin Area Spatial Extent

VR039 - Extinguished Lot and Admin Area Consistency

VR040 - Address in Vicmap Address

VR041 - Title Reference in VOTS

VR042 - Parcel Name Format

VR043 - Created Parcels for Cancelled Parcels

VR044 - Road Name in Vicmap Transport

$*$

$*$

$*$

$*$

$*$

$*$

$*$

$*$

*

$*$

$*$

$*$

$*$

$*$

$*$

$*$

$*$

$*$

$*$

$*$

$*$

$*$

$*$

$*$

$*$

$*$

$*$

* *

$*$

*

*

$*$

$*$

$*$

$*$

*

* 


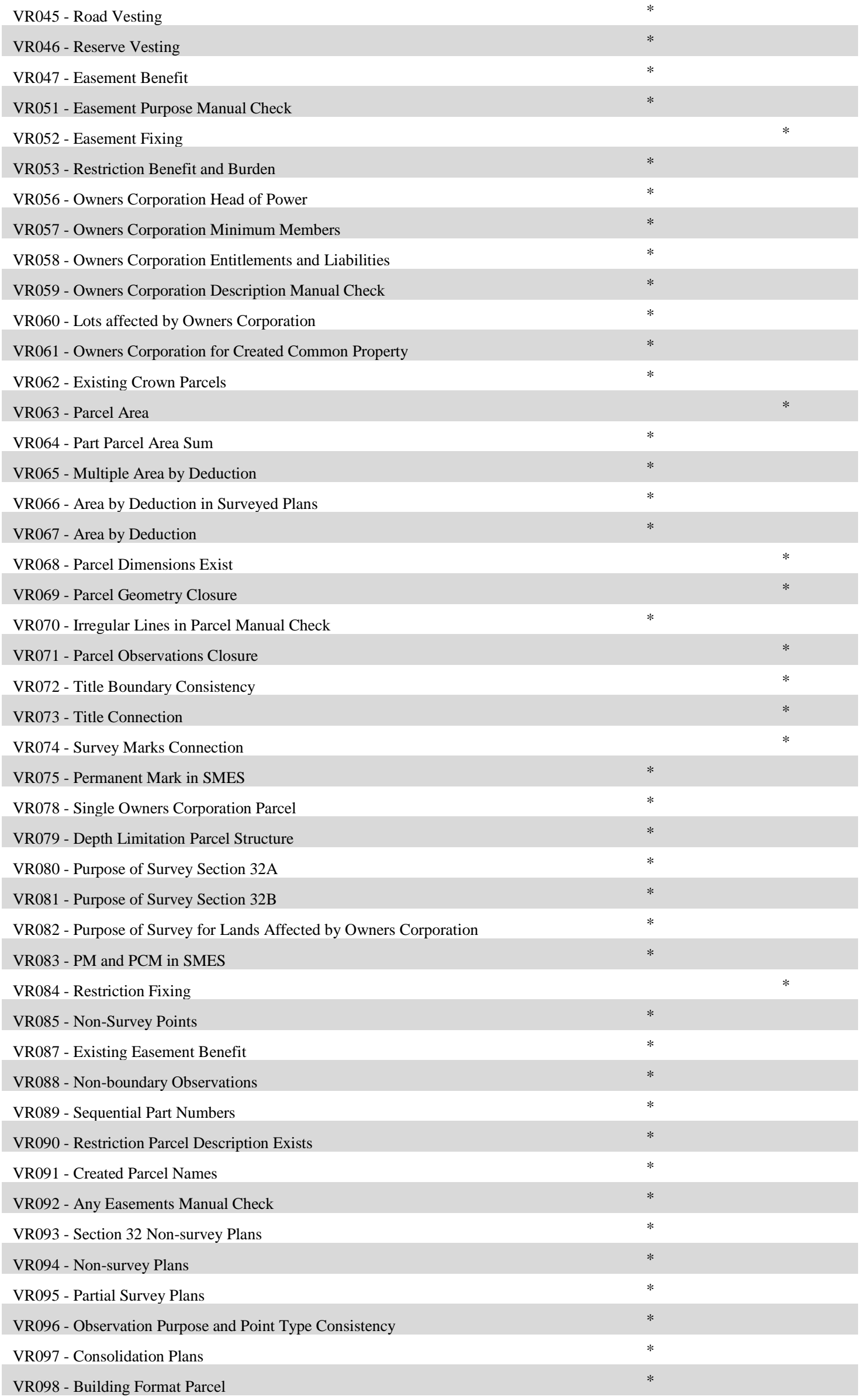




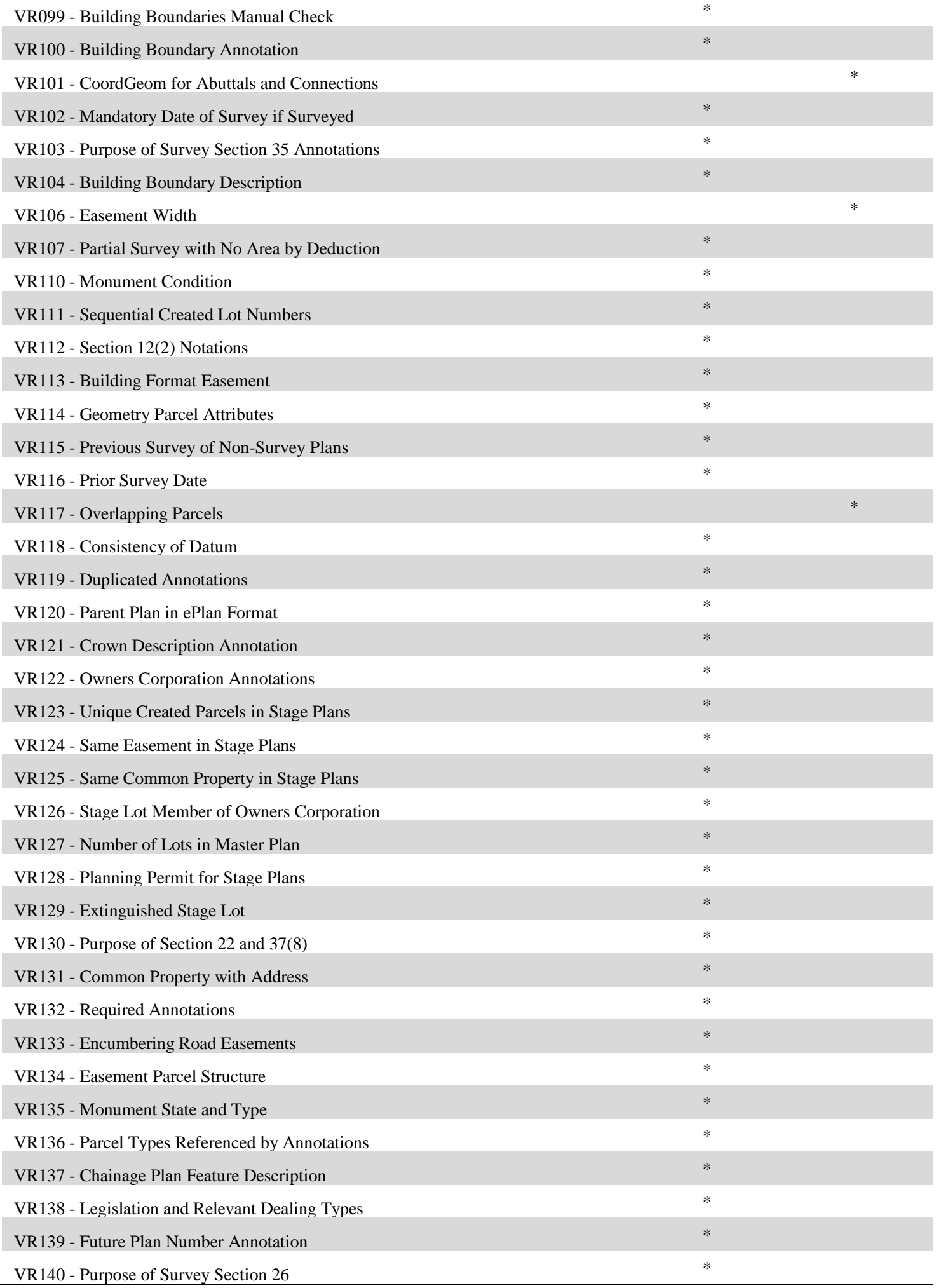




\section{University Library}

\section{- M M N E R VA A gateway to Melbourne's research publications}

Minerva Access is the Institutional Repository of The University of Melbourne

Author/s:

Shojaei, D;Olfat, H;Faundez, SIQ;Kalantari, M;Rajabifard, A;Briffa, M

Title:

Geometrical data validation in 3D digital cadastre: A case study for Victoria, Australia

Date:

2017-11-01

Citation:

Shojaei, D., Olfat, H., Faundez, S. I. Q., Kalantari, M., Rajabifard, A. \& Briffa, M. (2017).

Geometrical data validation in 3D digital cadastre: A case study for Victoria, Australia. Land Use Policy: the international journal covering all aspects of land use, 68, pp.638-648. https:// doi.org/10.1016/j.landusepol.2017.08.031.

Persistent Link:

http://hdl.handle.net/11343/245477 